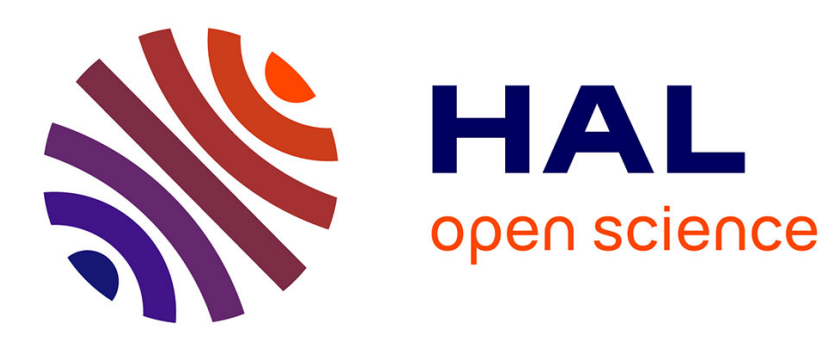

\title{
A PTIME-Complete Matching Problem for SLP-Compressed Words
}

\author{
Nicolas Markey, Philippe Schnoebelen
}

\section{To cite this version:}

Nicolas Markey, Philippe Schnoebelen. A PTIME-Complete Matching Problem for SLP-Compressed Words. Information Processing Letters, 2004, 90 (1), pp.3-6. 10.1016/j.ipl.2004.01.002 . hal01194622

\section{HAL Id: hal-01194622 \\ https://hal.science/hal-01194622}

Submitted on 7 Sep 2015

HAL is a multi-disciplinary open access archive for the deposit and dissemination of scientific research documents, whether they are published or not. The documents may come from teaching and research institutions in France or abroad, or from public or private research centers.
L'archive ouverte pluridisciplinaire HAL, est destinée au dépôt et à la diffusion de documents scientifiques de niveau recherche, publiés ou non, émanant des établissements d'enseignement et de recherche français ou étrangers, des laboratoires publics ou privés. 


\title{
A PTIME-complete matching problem for SLP-compressed words
}

\author{
N. Markey ${ }^{\mathrm{a}, \mathrm{b}} \mathrm{Ph}$. Schnoebelen ${ }^{\mathrm{b}}$ \\ a Département d'Informatique \\ Université Libre de Bruxelles \\ ${ }^{\mathrm{b}}$ Lab. Spécification \&s Vérification \\ ENS de Cachan \& CNRS UMR 8643
}

\begin{abstract}
SLP-compressed words are words given by simple deterministic grammars called "straight-line programs". We prove that the problem of deciding whether an SLPcompressed word is recognized by a FSA is complete for polynomial-time.
\end{abstract}

Key words: Algorithms, pattern-matching, compressed strings, complexity.

\section{Introduction}

Data compression is a powerful and versatile (hence popular) technique for reducing storage space. The issue of finding efficient algorithms working directly on compressed data received a lot of attention recently. In this field, a paradigmatic family of problems are (searching and) matching problems on compressed strings [10]. This is because strings are the most basic data structure, while searching and matching are ubiquitous problems.

For strings, many compression schemes exist [11]. At a conceptual level, the main compression schemes are SLP (for "Straight-Line Programs"), LZ (for "Lempel-Ziv"), RLZ (for "Restricted Lempel-Ziv") and LZW (Lempel-ZivWelch). These four schemes allow exponential compression: compressed strings may denote full-length texts of exponential length. They are more or less equivalent since it is possible to translate in polynomial-time compressed strings

Email addresses: nmarkey@ulb.ac.be (N. Markey), phs@lsv.ens-cachan.fr (Ph. Schnoebelen). 
from one scheme to another. Hence these compression schemes are successful on the same instances, and polynomial-time algorithms for one scheme can be transfered to the others.

Many matching problems on compressed strings can be solved in polynomialtime [9]. But there are situations where polynomial-time is not good enough: since compressed texts can be quite large, it is interesting to have efficient parallel algorithms (i.e., algorithms in $\mathrm{NC}$ ) or polynomial-time algorithms that only use polylog-space (i.e., in SC) ${ }^{1}$.

It has recently been observed that some matching problems on compressed strings admit $\mathrm{NC}$ algorithms $[5,4,6]$. However $\mathrm{NC}$ or $\mathrm{SC}$ algorithms are not always possible ${ }^{2}$, and some matching problems on compressed strings are known to be complete for polynomial-time $[3,4]$.

When it comes to algorithms in NC or SC, the three main compression schemes (SLP, LZ and LZW) are not equivalent. Regarding complexity below PTIME, the available results are scarce. Some problems are PTIME-hard for LZ $[5,9,4]$ but not for SLP. The evidence seems to indicate that SLP-compressed words are easier (that is, more amenable to efficient algorithms) than (R)LZ(W)compressed words.

In this note, we prove that deciding whether an SLP-compressed word is accepted by a (fixed) finite-state automaton (a FSA) is PTIME-complete. This is the first example of a PTIME-hard problem for SLP-compressed words.

We also consider matching problems simpler than FSA-acceptance, namely telling whether a given string occurs in the SLP-compressed word. For these problems, we provide simple proofs showing they admit LOGCFL algorithms, hence are low inside NC.

\section{Preliminaries}

We follow [9]. A straight-line program, or SLP, is a context-free grammar where the non-terminals $N_{1}, \ldots, N_{m}$ are ordered ( $N_{m}$ being the axiom), and where every non-terminal has a single production of the form $N_{i} \rightarrow a$ for a terminal $a$, or $N_{i} \rightarrow N_{j} N_{k}$ for some $j, k<i$. For an SLP $P$, we write $w\left(N_{i}\right)$ for the unique word described by $N_{i}$. Then $w(P)$ stands for $w\left(N_{m}\right)$.

\footnotetext{
$\overline{1}$ We refer to [8] or [7] for more details on classes below PTIME.

2 Here and in the following we implicitly make the standard assumption that PTIME does not collapse to NC or SC.
} 
Proposition 1 [9] Saying whether $w(P)$ is recognized by $\mathcal{A}$ (for $P$ an $S L P$, and $\mathcal{A}$ a finite-state automaton) can be done in time $O\left(|P| \times|\mathcal{A}|^{3}\right)$.

PROOF. [9] describes a simple dynamical programming solution. For two states $r, s$ of $\mathcal{A}$ and non-terminal $N_{i}$ of $P$, set $T[r, s, i]=$ true iff $w\left(N_{i}\right)$ labels a path going from $r$ to $s$ in $\mathcal{A}$. Obviously, if $N_{i} \rightarrow N_{j} N_{k}$ is a rule in $P$, then $T[r, s, i]=\bigvee_{u} T[r, u, j] \wedge T[u, s, k]$. Hence the table $T[\ldots]$ is easy to fill. Then we can use $T[\ldots]$ to see whether $w(P)$, i.e. $w\left(N_{m}\right)$, labels an accepting path.

\section{The main result}

Theorem 2 Saying whether $w(P)$ is accepted by a FSA $\mathcal{A}$, is PTIME-complete. Furthermore, PTIME-hardness already occurs for a fixed FSA.

In view of Proposition 1, only the second part of Theorem 2 has to be proved.

For this, we start by describing $\mathcal{A}_{5}$, the fixed FSA, and some of its properties. We were inspired by [1] for this construction. $\mathcal{A}_{5}$ has 5 states numbered from 0 to 4 and is depicted in Fig. 1. The initial state is 0 and the only final state is 1 .

For two states $s, t \in\{0,1,2,3,4\}$, we write $s \stackrel{a}{\rightarrow} t(s \stackrel{b}{\rightarrow} t, s \stackrel{c}{\rightarrow} t)$ when there is an $a$-labeled (resp., $b$-labeled, $c$-labeled) arrow from $s$ to $t$. We write $s \stackrel{\text { inc }}{\longrightarrow} t$ when $t=s+1 \bmod 5$ : hence $\stackrel{\text { inc }}{\longrightarrow}$ rotates one step clockwise. Observe that the relations $\stackrel{a}{\rightarrow}, \stackrel{b}{\rightarrow}, \stackrel{c}{\rightarrow}$, and $\stackrel{\text { inc }}{\longrightarrow}$ are in fact bijections. Further observe that $\stackrel{\text { inc }}{\longrightarrow}=\stackrel{b}{\rightarrow} \stackrel{a}{\rightarrow}$ (we compose functions from left to right: $f . f^{\prime}$ denotes $\left.f^{\prime} \circ f\right)$. Finally, we let $i d$ denote the identity between states.

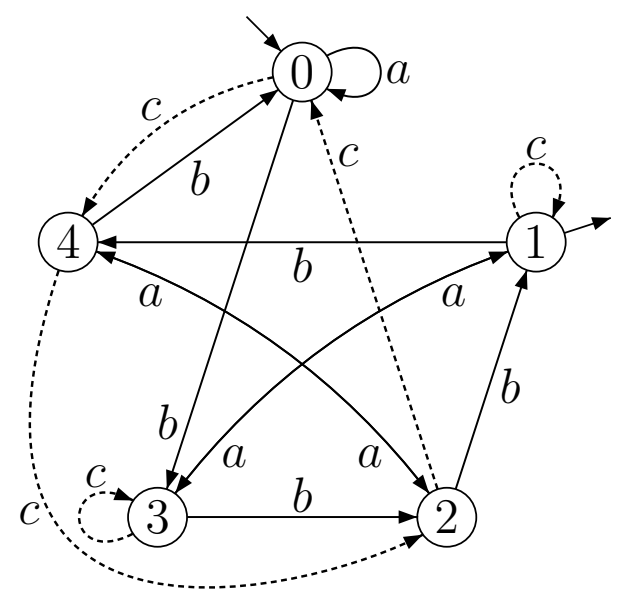

Fig. 1. $\mathcal{A}_{5}$, a fixed FSA. 
As the reader will easily check, the construction of $\mathcal{A}_{5}$ ensures that the following equalities hold:

$$
\begin{aligned}
& \stackrel{a}{\rightarrow} \cdot \stackrel{b}{\rightarrow} \cdot \stackrel{c}{\rightarrow} \cdot \stackrel{b}{\rightarrow} \cdot \stackrel{b}{\rightarrow}=\stackrel{\text { inc }}{\rightarrow} \\
& \stackrel{a}{\rightarrow} \cdot \stackrel{b}{\rightarrow} \stackrel{\text { inc }}{\rightarrow} \stackrel{c}{\rightarrow} \cdot \stackrel{b}{\rightarrow} \stackrel{\text { inc }}{\rightarrow} \stackrel{b}{\rightarrow}=\stackrel{\text { inc }}{\longrightarrow} \\
& \stackrel{a}{\rightarrow} \stackrel{\text { inc }}{\longrightarrow} \stackrel{b}{\rightarrow} \cdot \stackrel{c}{\rightarrow} \stackrel{\text { inc }}{\longrightarrow} \stackrel{b}{\rightarrow} \cdot \stackrel{b}{\rightarrow}=\stackrel{\text { inc }}{\longrightarrow} \\
& \stackrel{a}{\longrightarrow} \stackrel{\text { inc }}{\longrightarrow} \stackrel{b}{\longrightarrow} \stackrel{\text { inc }}{\longrightarrow} \stackrel{c}{\longrightarrow} \stackrel{\text { inc }}{\longrightarrow} \stackrel{b}{\longrightarrow} \stackrel{\text { inc }}{\longrightarrow} \stackrel{b}{\longrightarrow}=i d
\end{aligned}
$$

We now prove Theorem 2 by reduction from NAND-CIRCUIT-VALUE. Let $\mathcal{C}$ be a Boolean circuit made of nand gates with fan-in 2, some constant inputs, and with one designated output gate $g$. Fig. 2 displays an example.

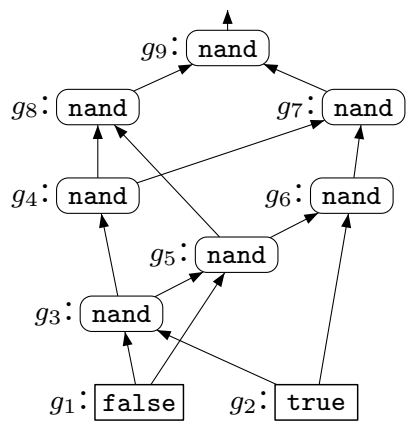

Fig. 2. $\mathcal{C}$, a Boolean circuit of nand gates.

Every gate $g_{i}$ evaluates to some $v\left(g_{i}\right) \in\{$ true, false $\}$ in the obvious way, and we let $v(\mathcal{C})$ denote the value of the output gate. It is well-known that telling whether $v(\mathcal{C})=$ true is a PTIME-complete problem [7, problem A.1.5].

With a circuit like $\mathcal{C}$, we associate a grammar $P_{\mathcal{C}}$ that has one non-terminal $N_{i}$ for every gate $g_{i}$. The rule for $N_{i}$ depends on whether $g_{i}$ is a nand gate inputing from $g_{j}$ and $g_{k}$ (note that $j=k$ is possible) or it is an input gate carrying true or false:

$$
\begin{array}{ll}
\text { if } g_{i}:=\operatorname{nand}\left(g_{j}, g_{k}\right) & \text { then } N_{i} \rightarrow a N_{j} b N_{k} c N_{j} b N_{k} b \\
\text { if } g_{i}:=\text { true } & \text { then } N_{i} \rightarrow b a \\
\text { if } g_{i}:=\text { false } & \text { then } N_{i} \rightarrow \varepsilon
\end{array}
$$

Finally, if $g_{m}$ is the output gate in $\mathcal{C}$, then the axiom of $P_{\mathcal{C}}$ is $N_{m} \cdot{ }^{3}$

For a word $w=a_{1} \ldots a_{m}$, we write $\stackrel{w}{\rightarrow}$ for the composition $\stackrel{a_{1}}{\rightarrow} \ldots \stackrel{a_{m}}{\rightarrow}$ (with $\stackrel{\varepsilon}{\rightarrow}$ being $i d)$.

3 Strictly speaking $P_{\mathcal{C}}$ is not a SLP, but it is easy to provide an equivalent linear-sized SLP. In particular, Eq. (4) needs not be used in view of false = nand(true, true). 
Lemma 3 For every gate $g_{i}$ in $\mathcal{C}$ :

(i) if $v\left(g_{i}\right)=$ true then $\stackrel{w\left(N_{i}\right)}{\longrightarrow}=\stackrel{\text { inc }}{\longrightarrow}$,

(ii) if $v\left(g_{i}\right)=$ false then $\stackrel{w\left(N_{i}\right)}{\longrightarrow}=i d$.

PROOF. By induction over the height of the gate in the circuit. For the base cases Eqs. (3) and (4) directly ensure (i) and (ii) (recall that $\stackrel{b a}{\rightarrow}=\stackrel{\text { inc }}{\rightarrow}$ ).

For the inductive step, $g_{i}$ is some nand $\left(g_{j}, g_{k}\right)$. There are four cases. Assume for example that $v\left(g_{j}\right)=$ true and $v\left(g_{k}\right)=$ false, so that $v\left(g_{i}\right)=$ nand $($ true, false $)=$ true and we have to prove $\stackrel{w\left(N_{i}\right)}{\longrightarrow}=\stackrel{\text { inc }}{\longrightarrow}$. By ind. hyp. $\stackrel{w\left(N_{j}\right)}{\longrightarrow}=\stackrel{\text { inc }}{\longrightarrow}$ and $\stackrel{w\left(N_{k}\right)}{\longrightarrow}=i d$. Now equation $(1 \mathrm{c})$ exactly states that $\stackrel{w\left(N_{i}\right)}{\longrightarrow}=\stackrel{\text { inc }}{\longrightarrow}$, given that $N_{i}$ is defined by (2). The three other cases use the other equalities in (1).

Corollary $4 v(\mathcal{C})=$ true iff $\stackrel{w\left(P_{\mathcal{C}}\right)}{\longrightarrow}=$ inc iff $w\left(P_{\mathcal{C}}\right)$ is accepted by $\mathcal{A}_{5}$.

Thus we have provided a logspace reduction from NAND-CIRCUIT-VALUE to acceptance of SLP compressed words by the fixed FSA $\mathcal{A}_{5}$, proving the second part of Theorem 2. $\mathcal{A}_{5}$ uses three letters for clarity but a two-letter alphabet would have been sufficient in view of $\stackrel{c}{\longrightarrow}=\stackrel{b b a b a b a b}{\longrightarrow}$.

\section{Efficient pattern-matching for SLP-compressed words}

In this section we look at pattern-matching problems that are special cases of FSA acceptance, trying to strengthen our PTIME-hardness result by extending it to a problem simpler than FSA acceptance.

It turns out that the problems we consider are in LOGDCFL and LOGCFL respectively, hence they are in $\mathrm{AC}^{1}$ and admit fast parallel algorithms. Note that these same problems are PTIME-complete for LZ-compressed words [4]. We see this as evidence that SLP-compressed words are "more manageable" than LZ-compressed words even though they are not significantly longer.

We start with the simplest matching problem: does $w(P)$ exactly match a given string $p$ ?

Theorem 5 Deciding whether $w(P)$ equals a string $p$ is in LOGDCFL.

PROOF. [Sketch] We show that the problem can be solved by a deterministic 
Turing Machine having access to an auxiliary unbounded pushdown storage and working in logarithmic space and polynomial time. Then we conclude relying on the characterization LOGDCFL $=\operatorname{AuxPD-DSPACE}(\log n, \operatorname{pol} n)$ from $[2,12]$.

The algorithm works as follows: Given an SLP $P$, one puts the axiom $N_{m}$ on the initially empty stack, and stores a pointer to the beginning of $p$. The stack will be used to store the sequence of non-terminals that remain to be developed. As long as the stack is not empty, we pop the first non-terminal, $N_{i}$ (say), from the top of the stack: either $P$ has a rule $N_{i} \rightarrow N_{j} N_{k}$, and we add $N_{k}$ and $N_{j}$, in that order, onto the stack; or the rule is $N_{i} \rightarrow a$, and we check that $a$ is the current letter in $p$, in which case we advance the pointer inside $p$.

The algorithm runs in time $O(|p| \cdot|P|)$, and needs space $O(\log |p|+\log |P|)$ to memorize the pointers inside $p$ and inside $P$.

Next we consider combined patterns of the form $p_{0} \star p_{1} \star \cdots \star p_{m}$ where the $p_{i}$ are words and where $\star$ means "any substring". Such combined patterns can express problems like "does $p$ occur inside the text?" (by picking $m=2$, $p_{0}=p_{m}=\varepsilon$ and $p_{1}=p$ ), and "is $p$ a subword of the text?" (for $p$ of the form $a_{1} \ldots a_{n}$, one picks $m=n+1, p_{0}=p_{m}=\varepsilon$ and $p_{i}=a_{i}$ for $\left.1 \leq i \leq n\right)$.

Theorem 6 Deciding whether $w(P)$ matches a combined pattern $p_{0} \star \cdots \star p_{m}$ is in LOGCFL.

PROOF. [Sketch] We proceed as in the previous Theorem, as if we were checking that $w(P)$ equals $p_{0} \star \cdots \star p_{m}$. The difference is that, when we are matching $\mathrm{a} \star$ inside the pattern, we just discard letters from $w(P)$. This is achieved by nondeterministically guessing the (occurrences of) non-terminals we won't have to develop when we pop them from the stack. Note that this runs in polynomial-time since we can discard a useless (occurrence of a) nonterminal as soon as possible, i.e. before expanding it. (Expanding it and discarding its expansion would require exponential-time.) One concludes with the characterization LOGCFL $=\mathbf{A u x P D - N S P A C E}(\log n, \operatorname{pol} n)$ from $[2,12]$.

\section{References}

[1] M. Beaudry, P. McKenzie, P. Péladeau, and D. Thérien. Finite monoids: From word to circuit evaluation. SIAM J. Computing, 26(1):138-152, 1997.

[2] S. A. Cook. Characterizations of pushdown machines in terms of time-bounded computers. Journal of the ACM, 18(1):4-18, 1971. 
[3] S. De Agostino. P-complete problems in data compression. Theoretical Computer Science, 127(1):181-186, 1994.

[4] L. Gąsieniec, A. Gibbons, and W. Rytter. Efficiency of fast parallel patternsearching in highly compressed texts. In Proc. 24th Int. Symp. Math. Found. Comp. Sci. (MFCS'99), Szklarska Poreba, Poland, Sep. 1999, volume 1672 of Lecture Notes in Computer Science, pages 48-58. Springer, 1999.

[5] L. Gąsieniec, M. Karpinski, W. Plandowski, and W. Rytter. Efficient algorithms for Lempel-Zip encoding. In Proc. 5th Scandinavian Workshop on Algorithm Theory (SWAT'96), Reykjavik, Iceland, July 1996, volume 1097 of Lecture Notes in Computer Science, pages 392-403. Springer, 1996.

[6] L. Gąsieniec and W. Rytter. Almost optimal fully LZW-compressed pattern matching. In Proc. Data Compression Conference (DCC'99), Mar. 1999, Snowbird, Utah, USA, pages 316-325. IEEE Comp. Soc. Press, 1999.

[7] R. Greenlaw, H. J. Hoover, and W. L. Ruzzo. Limits to Parallel Computation: P-Completeness Theory. Oxford Univ. Press, 1995.

[8] D. S. Johnson. A catalog of complexity classes. In J. van Leeuwen, editor, Handbook of Theoretical Computer Science, volume A, chapter 2, pages 67-161. Elsevier Science, 1990.

[9] W. Plandowski and W. Rytter. Complexity of language recognition problems for compressed words. In J. Karhumaki, H. Maurer, G. Păun, and G. Rozenberg, editors, Jewels are Forever, pages 262-272. Springer, 1999.

[10] W. Rytter. Algorithms on compressed strings and arrays. In Proc. 26th Conf. Current Trends in Theory and Practice of Informatics (SOFSEM'99), Milovy, Czech Republic, Nov. 1999, volume 1725 of Lecture Notes in Computer Science, pages 48-65. Springer, 1999.

[11] J. A. Storer and T. G. Szymanski. Data compression via textual substitution. Journal of the ACM, 29(4):928-951, 1982.

[12] I. H. Sudborough. On the tape complexity of deterministic context-free languages. Journal of the ACM, 25(3):405-414, 1978. 